BMJ Open

Diabetes

Research

\& Care

\title{
Incidence and associates of diabetic ketoacidosis in a community-based cohort: the Fremantle Diabetes Study Phase II
}

\author{
Timothy M E Davis (D), Wendy Davis
}

\begin{abstract}
To cite: Davis TME, Davis W. Incidence and associates of diabetic ketoacidosis in a community-based cohort: the Fremantle Diabetes Study Phase II. BMJ Open Diab Res Care 2020;8:e000983. doi:10.1136/ bmjdrc-2019-000983
\end{abstract}

Received 15 October 2019 Revised 22 December 2019 Accepted 18 February 2020

Check for updates

(c) Author(s) (or their employer(s)) 2020. Re-use permitted under CC BY-NC. No commercial re-use. See rights and permissions. Published by BMJ.

Medical School, University of Western Australia, Perth, Western Australia, Australia

Correspondence to Dr Timothy M E Davis; tim.davis@uwa.edu.au

\section{ABSTRACT}

Objective To assess the incidence and associates of diabetic ketoacidosis (DKA) in a representative communitybased cohort.

Methods All hospitalizations of 1724 participants in the Fremantle Diabetes Study Phase II for/with DKA (plasma glucose $>13.8 \mathrm{mmol} / \mathrm{L}$, urinary $/$ serum ketones, serum bicarbonate $<18 \mathrm{mmol} / \mathrm{L}$ and/or arterial/venous $\mathrm{pH}<7.30$ ) were identified between study entry from 2008 to 2011 and end-2013. Details of each episode were categorized by chart review as confirmed/probable DKA, possible DKA or not DKA. Incidence rates by diabetes type were calculated. Cox proportional hazards modeling determined predictors of first episode, and negative binomial regression identified predictors of frequency.

Results There were 53 coded DKA episodes (41 first episodes, 12 recurrences), of which 19 (35.8\%) were incorrectly coded, 9 (17.0\%) had possible DKA and 25 (47.2\%) had confirmed/probable DKA. Of this latter group, $44 \%$ had type 1 diabetes, $32 \%$ had type 2 diabetes, $12 \%$ had latent autoimmune diabetes of adults (LADA) and $12 \%$ had secondary diabetes. The overall incidence of confirmed/probable DKA ( $95 \% \mathrm{Cl}$ ) was $35.6(23.0$ to 52.6)/10 000 person-years (178.6 (85.7 to 328.5)/10 000 person-years for type 1 diabetes, 13.3 (5.7 to 26.1$) / 10$ 000 person-years for type 2 diabetes, 121.5 (33.1 to 311.0)/10 000 person-years for LADA and 446.5 (92.1 to 1304.9)/10 000 person-years for secondary diabetes). Baseline In(fasting serum C-peptide) (inversely), glycated hemoglobin and secondary diabetes predicted both incident first confirmed/probable DKA episode and the frequency of DKA $(p<0.001)$.

Conclusions These data highlight the contribution of poor glycemic control and limited pancreatic beta cell function to incident DKA, and show that people with types of diabetes other than type 1 , especially secondary diabetes, are at risk.

\section{INTRODUCTION}

Diabetic ketoacidosis (DKA) is an acute metabolic complication of diabetes mellitus that, if not promptly recognized and treated, can

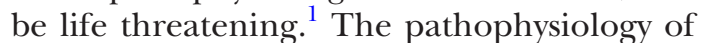
DKA is characterized by insulin deficiency in concert with increased counter-regulatory hormone secretion and peripheral insulin

\section{Significance of this study}

What is already known about this subject?

- Diabetic ketoacidosis is a serious acute metabolic complication of diabetes that can affect people with types of diabetes other than type 1 .

What are the new findings?

- In community-based people with well-characterized diabetes presenting with diabetic ketoacidosis, the minority had type 1 diabetes; type 2 diabetes, latent autoimmune diabetes of adults and secondary diabetes, but not maturity onset diabetes of the young, were also represented.

How might these results change the focus of research or clinical practice?

- Diabetic ketoacidosis should be considered in the differential diagnosis of metabolic decompensation in all types of diabetes.

- Although it remains an uncommon acute complication of diabetes, diabetic ketoacidosis occurs in types of diabetes other than type 1.

- Poor glycemic control and limited pancreatic beta cell function are important predisposing factors while the risk of diabetic ketoacidosis could be underestimated in people with secondary diabetes.

- Reliance on administrative data without individual patient chart review could overestimate the incidence of diabetic ketoacidosis, with implications for health service planning and delivery.

resistance, resulting in hyperglycemia, ketosis, dehydration and electrolyte imbalance. ${ }^{2}$ DKA has been conventionally associated with type 1 diabetes but stressors including trauma and infection can increase the risk of DKA in other forms of diabetes. In recent series of hospitalized patients, type 2 diabetes accounted for up to around a half of all DKA cases. ${ }^{3-6}$ The incidence of DKA in people with latent autoimmune diabetes of adults (LADA) has assumed to be very low because of relative preservation of insulin secretion compared with type 1 diabetes. ${ }^{7}$ However, although 
DKA early in the course of autoimmune diabetes infers a diagnosis of type 1 rather than LADA, ${ }^{8}$ this phenotypic distinction is no longer regarded as important ${ }^{9}$ and is, in any case, lost over time as pancreatic beta cell function declines in LADA patients. Patients with genetic mutations associated with maturity onset diabetes of the young (MODY) and neonatal diabetes have a very low risk of DKA. ${ }^{10}$ It has long been recognized that secondary diabetes can be complicated by DKA even though there may be limited glucagon secretion as part of the counterregulatory response. ${ }^{11}$

Characterization of the incidence of DKA has important implications for health service planning and delivery, as well as being an indirect marker of the overall quality of local glycemic management. ${ }^{12}$ However, reliance on administrative hospital data to ascertain cases of DKA risks inaccurate estimates through miscoding. ${ }^{6}$ In addition, errors in classification of type of diabetes, even as simply type 1 or type $2,{ }^{6}{ }^{13}$ can have clinical implications since there is some evidence that the management of DKA should be tailored to the underlying diabetes type. ${ }^{5}$ In light of these considerations, we have assessed the incidence and associates of first health service attendance for DKA ascertained from patient records in a well-characterized and representative community-based cohort of people across the spectrum of diabetes types.

\section{MATERIALS AND METHODS}

\section{Participants}

The Fremantle Diabetes Study Phase II (FDS2) is an ongoing longitudinal observational study carried out in a postcode-defined geographical area surrounding the port city of Fremantle in Western Australia (WA). ${ }^{14} \mathrm{We}$ identified 4639 people with diabetes (excluding gestational diabetes) during FDS2 registration between 2008 and 2011 with 1668 (36\%) being recruited. A further 64 surviving participants recruited to the first phase of FDS between 1993 and 1996 and who lived outside the study area when FDS2 started were also recruited. All subjects gave written informed consent.

\section{Clinical assessment}

All FDS2 participants were scheduled for detailed face-toface assessments at entry and then biennially, interspersed with biennial postal questionnaires. ${ }^{14}$ Face-to-face assessments included a standardized comprehensive questionnaire, physical examination and fasting biochemical tests performed in a single nationally accredited laboratory. Ethnicity was categorized based on self-selection, country/ countries of birth, and parents' birth and grandparents' birth and language(s) spoken at home as Anglo-Celt, Southern European, Other European, Asian, Aboriginal or mixed/other. Complications were identified using standard definitions. ${ }^{14}$ Microalbuminuria was defined as a urine albumin:creatinine ratio $\geq 3.0 \mathrm{mg} / \mathrm{mmol}$ and renal impairment assessed from the estimated glomerular filtration rate (eGFR). ${ }^{15}$ Neuropathy was defined using the clinical portion of the Michigan Neuropathy Screening Instrument. ${ }^{16}$ Retinopathy was defined as one microaneurysm in either eye or worse and/or evidence of previous laser treatment on fundus photography, and/ or more detailed ophthalmological data in those assessed for photocoagulation.

Type of diabetes (type 1, type 2, LADA, monogenic or secondary) was ascertained at study entry based on diabetes treatment history (especially insulin and its initiation relative to diagnosis), body mass index (BMI), age at diagnosis, nature of first presentation and/or selfidentification including a previous clinician diagnosis of MODY or neonatal diabetes. Case records consulted for evidence of ketonemia, as well as autoantibodies, serum insulin and/or C-peptide concentrations, and genotyping, if available. In addition, all participants aged $<35$ years at diagnosis were assessed using the UK MODY risk prediction models and those identified as probable MODY underwent subsequent genetic testing for relevant mutations. Serum glutamic acid decarboxylase (GAD65) autoantibody titers were determined for all participants with clinically-diagnosed type 2 diabetes using a commercially available ELISA kit, with GAD65 positivity, and thus a diagnosis of LADA, defined as a titer $\geq 5 \mathrm{U} / \mathrm{mL}^{17}$

\section{Ascertainment of diabetic ketoacidosis}

Episodes of care for DKA using International Classification of Disease (ICD)-9 CM and ICD-10-AM codes were identified between study entry from 2008 to 2011 and end-2013 from three health service resources that are part of the WA Data Linkage System (WADLS) ${ }^{18}$ : i) public or private hospitalizations, ii) emergency department presentations and iii) attendances documented in the St John's Ambulance Database. For each episode, the case notes were assessed by an experienced clinician (TMED) for evidence of DKA which was defined as a plasma glucose $>13.8 \mathrm{mmol} / \mathrm{L}$, ketones in serum or urine and a serum bicarbonate $<18 \mathrm{mmol} / \mathrm{L}$ and/or arterial/venous $\mathrm{pH}<7.30$. Each episode was categorized as confirmed (satisfying all criteria), probable (satisfying the criteria but with no documented ketone measurement, and no other cause for acidosis with or without similar prior hospitalizations), possible (incomplete biochemical data with or without another plausible cause for acidosis such as acute renal impairment) or not DKA (sufficient biochemical data to exclude the diagnosis despite being coded as DKA).

\section{Data analysis}

Statistical analyses were performed using IBM SPSS for Windows (V.25.0, IBM, Armonk, New York, USA) and Stata (V.15.1, StataCorp, College Station, Texas, USA). Data are presented as proportions, mean $\pm \mathrm{SD}$, geometric mean (SD range) or median (IQR). For independent samples, two-way comparisons for categorical variables were by Fisher's exact test, for normally or log-normally distributed continuous variables by independent samples t-test and for variables not conforming to normal or log 
(ln)-normal distribution by Mann-Whitney U test. A twotailed significance level of $\mathrm{p}<0.05$ was used. To determine independent predictors of time to first episode of confirmed/probable DKA during follow-up, clinically plausible baseline variables with $\mathrm{p}<0.05$ in bivariate analyses, with multiple imputed $(\times 20)$ if there were missing values, were included in Cox proportional hazards modeling. Variable entry was set at $\mathrm{p}<0.01$ and removal $p \geq 0.01$ due to the low number of outcomes. Predictors of the frequency of DKA in participants with multiple episodes were assessed using negative binomial regression, the imputed dataset and the same strict entry/ removal criteria. To allow for the skewed distribution of duration of follow-up, an offset variable of the natural logarithm (ln) of the total follow-up time for each participant was used.

\section{RESULTS}

\section{Baseline participant characteristics}

The FDS2 cohort of 1732 participants had a mean \pm SD age of $63.8 \pm 13.6$ years at study entry, $52.1 \%$ were males, median (IQR) diabetes duration $10.0(3.0-16.8)$ years, and $85.6 \%$ had type 2 diabetes, $8.0 \%$ type 1 diabetes, $4.9 \%$ LADA, $1.0 \%$ secondary diabetes and $0.5 \%$ monogenic diabetes. Most (54.3\%) were of Anglo-Celt ethnicity, $10.9 \%$ were current smokers and their median (IQR) alcohol consumption was $0.1(0-1.2)$ standards drinks (where 1 standard drink is equivalent to $10 \mathrm{~g}$ alcohol) per day. Seventeen participants $(1.0 \%)$ had one or more unvalidated hospitalizations for/with DKA in the 5 years prior to baseline. Four of these $(23.5 \%)$ had a subsequent validated incident DKA event.

\section{First episode of diabetic ketoacidosis during follow-up}

During a mean \pm SD $4.1 \pm 1.1$ years of follow-up to death or end-2013, there were 41 recorded episodes of first DKA during the follow-up period in FDS2 participants including one coroner-adjudicated death attributed to DKA. Of these 41 cases, 17 (41.5\%) were confirmed and $1(2.4 \%)$ probable based on clinical/laboratory data, representing a total of $18(43.9 \%)$ confirmed/probable cases. There were 16 cases $(39.0 \%)$ that were incorrectly coded as DKA and, in 4 of these who had subsequent additional coded DKA episodes during the period of follow-up, none of these hospitalizations met the criteria for DKA. A further seven participants (17.1\%; mean age $62.1 \pm 17.2$ years, $57.1 \%$ males) could not be categorized because of insufficient data and were not considered further in the present analysis.

Of the 18 confirmed/probable cases, $50.0 \%$ were in participants with type 1 diabetes, followed by $27.8 \%$ with type 2 diabetes, $11.1 \%$ with LADA and $11.1 \%$ with secondary diabetes. There were no cases of DKA in participants with monogenic diabetes. The overall incidence rate $(95 \% \mathrm{CI})$ for confirmed/probable first DKA (18 cases in 1725 FDS2 participants during 6982 personyears of follow-up to confirmed/probable first DKA, death or end-December 2013, whichever came first) was 25.8 (15.3 to 40.7$) / 10000$ person-years. When subdivided by diabetes type, the incidence rates were $8.3(2.7$ to 19.4$) / 10000$ person-years for type 2 diabetes, 166.4 (76.1 to 315.9)/10 000 person-years for type 1 diabetes, 61.4 (7.4 to 221.8)/10 000 person-years for LADA and 306.9 (37.2 to 1108.8) / 10000 person-years for secondary diabetes including the one fatal case.

The characteristics of FDS2 participants who did not experience an episode of DKA during follow-up and those of the 18 confirmed/probable cases are summarized in table 1. The confirmed/probable cases had a younger age at diagnosis, a longer diabetes duration and a lower BMI than those who did not develop DKA. They were more likely to have forms of diabetes other than type 2 apart from monogenic diabetes, and were more likely to be insulin-treated as a result. They also had a higher glycated hemoglobin (HbAlc), a lower serum C-peptide and a greater likelihood of prior severe hypoglycemia than those without DKA during follow-up. They were more likely to have at least stage 3 renal impairment.

\section{Predictors of first episode of diabetic ketoacidosis during follow-up}

Of the 1725 participants, $25(1.4 \%)$ had at least one missing value among the clinically plausible variables of interest. Age at diabetes diagnosis, BMI, HbAlc, serum C-peptide, insulin use and eGFR had between one and eight missing values that were imputed. In a Cox proportional hazards model, the independent predictors of time to first episode of DKA were the serum C-peptide (inversely), a diagnosis of secondary diabetes and HbA1c (all $\mathrm{p}<0.001$; table 2).

\section{Recurrent incident diabetic ketoacidosis events}

Eight participants $(0.5 \%$ or $44.4 \%$ of those with a first incident DKA episode during follow-up) had one or more further hospitalizations coded as DKA. Of these 12 additional events, 5 were confirmed in three participants and 2 were categorized as probable in two participants (58.3\% confirmed/probable cases) after chart review, 3 (25.0\%) were incorrectly coded as DKA and 2 (16.7\%) could not be categorized because of insufficient data. Of the five with recurrent confirmed/probable DKA, one had type 1 diabetes, two had type 2 diabetes, one had LADA and one had secondary diabetes.

When combined with the first DKA episodes, the overall percentages of the 25 confirmed/probable cases in 1725 FDS2 participants during 7022 person-years of follow-up to death or end-December 2013 (whichever came first), DKA cases by type of diabetes were $32 \%$ type 2, 44\% type 1, 12\% LADA and $12 \%$ secondary diabetes. The overall incidence rate $(95 \%$ CI) was 35.6 (23.0 to 52.6)/10 000 person-years. When subdivided by diabetes type, the overall incidence rates were 13.3 (5.7 to 26.1$) / 10000$ person-years for type 2 diabetes, 178.6 (85.7 to 328.5)/10 000 person-years for type 1 diabetes, 121.5 (33.1 to 311.0)/10 000 person-years for LADA and 
Table 1 Characteristics of Fremantle Diabetes Study Phase II participants who did not develop DKA during follow-up and those with a confirmed/probable episode

\begin{tabular}{|c|c|c|c|}
\hline & No DKA & Confirmed/probable DKA & $P$ value \\
\hline Number (\%) & 1707 & 18 & \\
\hline Age (years) & $63.9 \pm 13.5$ & $56.0 \pm 19.3$ & 0.10 \\
\hline Male (\%) & 52.0 & 55.6 & 0.82 \\
\hline Ethnic background (\%): Anglo-Celt & 54.5 & 33.3 & 0.08 \\
\hline Southern European & 11.9 & 5.6 & \\
\hline Other European & 7.0 & 16.7 & \\
\hline Asian & 4.2 & 0 & \\
\hline Aboriginal & 6.4 & 11.1 & \\
\hline Mixed/Other & 15.9 & 33.3 & \\
\hline Education (\%): primary or lower & 12.7 & 5.6 & 0.76 \\
\hline Secondary & 52.1 & 61.1 & \\
\hline Some/Completed tertiary & 35.2 & 33.3 & \\
\hline Age at diagnosis (years) & $52.9 \pm 15.1$ & $35.8 \pm 14.2$ & $<0.001$ \\
\hline Diabetes duration (years) ${ }^{*}$ & $10.0(3.0-16.6)$ & $17.4(9.3-28.9)$ & 0.007 \\
\hline Diabetes type (\%): type 1 & 7.4 & 50.0 & $<0.001$ \\
\hline LADA & 4.9 & 11.1 & \\
\hline Type 2 & 86.3 & 27.8 & \\
\hline Secondary & 0.9 & 11.1 & \\
\hline Monogenic & 0.5 & 0 & \\
\hline Diabetes treatment (\%): diet & 22.2 & 0 & $<0.001$ \\
\hline Oral agents \pm non-insulin injectables & 49.1 & 5.6 & \\
\hline Insulin \pm non-insulin injectables & 12.6 & 66.7 & \\
\hline Insulin+other agents & 16.1 & 27.8 & \\
\hline Smoking status (\%): never & 43.2 & 33.3 & 0.26 \\
\hline Ex-smoker & 46.1 & 44.4 & \\
\hline Current & 10.7 & 22.2 & \\
\hline Alcohol (standard drinks/day) ${ }^{\star}$ & $0.1(0-1.2)$ & $0.9(0-2.0)$ & 0.27 \\
\hline $\mathrm{BMI}\left(\mathrm{kg} / \mathrm{m}^{2}\right)$ & $30.8 \pm 6.2$ & $27.5 \pm 5.0$ & 0.023 \\
\hline Fasting serum glucose (mmol/L)* & $7.3(6.2-9.1)$ & $7.0(5.6-11.9)$ & 0.80 \\
\hline $\mathrm{HbA} 1 \mathrm{c}(\%)^{*}$ & $6.9(6.3-7.8)$ & $8.7(7.6-10.3)$ & $<0.001$ \\
\hline $\mathrm{HbA} 1 \mathrm{c}(\mathrm{mmol} / \mathrm{mol})^{*}$ & $52(45-62)$ & $72(60-89)$ & $<0.001$ \\
\hline Fasting serum C-peptide (pmol/L) & $168(38-741)$ & $11(2-67)$ & $<0.001$ \\
\hline Severe hypoglycemia history (\%) & 6.7 & 44.4 & $<0.001$ \\
\hline Any retinopathy (\%) & 39.7 & 62.5 & 0.08 \\
\hline Urinary albumin:creatinine $(\mathrm{mg} / \mathrm{mmol}))$ & $3.2(0.8-12.3)$ & $4.7(0.8-26.2)$ & 0.24 \\
\hline Peripheral sensory neuropathy (\%) & 56.4 & 61.1 & 0.81 \\
\hline eGFR (\%) $\geq 90 \mathrm{~mL} / \mathrm{min} / 1.73 \mathrm{~m}^{2}$ & 40.8 & 52.9 & 0.021 \\
\hline $60-89 \mathrm{~mL} / \mathrm{min} / 1.73 \mathrm{~m}^{2}$ & 42.7 & 17.6 & \\
\hline $45-59 \mathrm{~mL} / \mathrm{min} / 1.73 \mathrm{~m}^{2}$ & 8.6 & 5.9 & \\
\hline $30-44 \mathrm{~mL} / \mathrm{min} / 1.73 \mathrm{~m}^{2}$ & 5.2 & 23.5 & \\
\hline$<30 \mathrm{~mL} / \mathrm{min} / 1.73 \mathrm{~m}^{2}$ & 2.6 & 0 & \\
\hline
\end{tabular}

Data are percentages, mean $\pm \mathrm{SD}$, geometric mean (SD range) or ${ }^{*}$ median (IQR).

BMI, body mass index; DKA, diabetic ketoacidosis; eGFR, estimated glomerular filtration rate; HbA1c, glycated hemoglobin;

LADA, latent autoimmune diabetes of adults. 
Table 2 Independent baseline associates of time to first episode of diabetic ketoacidosis during follow-up

\begin{tabular}{lcc}
\hline & HR $(95 \%$ Cl) & P value \\
\hline $\begin{array}{l}\text { In(serum C-peptide } \\
\text { (pmol/L)) }\end{array}$ & $0.44(0.33$ to 0.59$)$ & $<0.001$ \\
Secondary diabetes & $22.1(4.82$ to 101$)$ & $<0.001$ \\
HbA1c (increase of $1 \%$ or & $1.76(1.41$ to 2.19$)$ & $<0.001$ \\
$7 \mathrm{mmol} / \mathrm{mol})$ & & \\
\hline
\end{tabular}

*A 2.72-fold increase in serum C-peptide corresponds to an increase of 1 in In(serum C-peptide).

$\mathrm{HbA1c}$, glycated hemoglobin.

446.5 (92.1 to 1304.9)/10 000 person-years for secondary diabetes including the one fatal case.

\section{Predictors of all incident episodes of diabetic ketoacidosis}

In an analysis of all confirmed/probable DKA epsiodes (first and recurrent during follow-up) and in a negative binomial model, $\ln$ (serum C-peptide) (inversely), a diagnosis of secondary diabetes, HbAlc and insulin treatment at study entry independently predicted DKA frequency (all $\mathrm{p}<0.001$; table 3).

\section{Diabetes management changes as a result of hospitalization with DKA}

All participants were reviewed by the multidisciplinary diabetes team during hospitalization. When there was both a clear precipitating factor (infection in many cases, but a faulty insulin pump in one and accidental omission of insulin treatment by nursing home staff in another) and the admission HbAlc indicated acceptable prior glycemic control, diabetes treatment regimens were not altered, but relevant information including that relating to sick day management was provided before discharge. Adherence was emphasized in those in which this was considered to be a contributing factor, and insulin regimens were intensified in those whose HbAlc was above an appropriate target. This included participants with type 2 diabetes, all of whom were insulin-treated at the time of their first and subsequent DKA episodes.

Table 3 Independent baseline associates of diabetic ketoacidosis frequency during follow-up

\begin{tabular}{|c|c|c|}
\hline & $\begin{array}{l}\text { Incident rate ratio } \\
(95 \% \mathrm{Cl})\end{array}$ & $P$ value \\
\hline $\begin{array}{l}\text { In(serum C-peptide } \\
(\mathrm{pmol} / \mathrm{L}))^{\star}\end{array}$ & 0.57 (0.42 to 0.77$)$ & $<0.001$ \\
\hline Secondary diabetes & 57.0 (6.06 to 536$)$ & $<0.001$ \\
\hline $\begin{array}{l}\mathrm{HbA} 1 \mathrm{c} \text { (increase of } 1 \% \text { or } \\
7 \mathrm{mmol} / \mathrm{mol} \text { ) }\end{array}$ & 1.76 (1.37 to 2.25$)$ & $<0.001$ \\
\hline Insulin use at study entry & 19.4 (4.22 to 89.4$)$ & $<0.001$ \\
\hline
\end{tabular}

${ }^{*} \mathrm{~A}$ 2.72-fold increase in serum C-peptide corresponds to an increase of 1 in $\ln ($ serum C-peptide).

$\mathrm{HbA1c}$, glycated hemoglobin.

\section{DISCUSSION}

The present study shows that DKA remains an uncommon acute metabolic complication of diabetes, with an overall incidence of $<4$ confirmed/probable cases per 1000 patient-years. Half of the first episodes of DKA during follow-up in our study were in participants with type 1 diabetes and about half of the remainder were in those with type 2 diabetes. Participants with LADA and secondary diabetes made up the balance of cases, but there were no episodes of DKA among the small number with monogenic diabetes. Multivariable analysis confirmed that chronically poor glycemic control and limited pancreatic beta cell reserve were strong independent predictors of first incident DKA, but secondary diabetes was also in the model. These three variables were also predictors of the frequency of DKA episodes, together with insulin treatment at study entry.

The reported incidence of DKA complicating type 1 diabetes from a recent systematic review is up to 560 cases per 10000 person-years ${ }^{19}$ and our overall incidence and $95 \%$ CIs of 178.6 (85.7 to 328.5) / 10000 person-years were within this range. Similarly, published rates for type 2 diabetes are up to $20 / 10000$ person-years,${ }^{20}$ which is also consistent with our estimate (13.3 (5.7 to 26.1)/10 000 person-years). Sodium glucose cotransporter 2 inhibitors (SGLT2Is) are a class of blood glucose-lowering agent introduced into Australia after the follow-up period used in the present study. They are associated with an increased incidence of DKA in type 2 diabetes in most observational studies if not in clinical trials, ${ }^{21}$ with approaching half of the cases presenting with a serum glucose lower than the $13.7 \mathrm{mmol} / \mathrm{L}$ conventionally required for diagnosis (so-called 'euglycemic' DKA). ${ }^{22}$ However, inconsistencies between studies including some population-based data showing no significant increase in risk ${ }^{23}$ suggest that factors influencing prescribing, such as recent warnings of high-risk situations for SGLTIassociated DKA and measures for its prevention such as in the perioperative period, ${ }^{25}$ may vary geographically.

We found that insulin treatment at study entry was independently predictive of the frequency of DKA. Since all participants were insulin-treated at the time of DKA hospitalization, this implies that longer duration insulin treatment may have a role in DKA risk in type 2 diabetes. $\mathrm{We}^{26}$ and others ${ }^{27}$ have found a relationship between duration of insulin therapy and risk of severe hypoglycemia in type 2 diabetes. Taken together, these observations highlight the potential for long duration diabetes treatment to lead to acute complications at the extremes of glycemia.

The overall incidence of first DKA complicating LADA in the present study (121.5/10 000 person-years) was between our estimates for type 1 and 2 diabetes (178.6 and 13.1/10 000 person-years, respectively), paralleling the intermediate pancreatic beta cell function that has been reported previously. ${ }^{28}$ Although we had relatively few participants with secondary diabetes (which was due mostly to chronic pancreatitis), the incidence of DKA in 
this group was the highest at 446.5/10 000 person-years and secondary diabetes was an independent predictor of both incident first confirmed/probable DKA and the frequency of DKA. There are no previously published comparative data, but the potential for DKA to complicate this form of diabetes has been recognized for some time, ${ }^{11}$ including the risk of death ${ }^{29}$ as in the case of one of our participants. As in the present study, patients with monogenic diabetes, with the exception of maternally inherited diabetes and deafness, ${ }^{30}$ have a very low risk of DKA because there is not the usually progressive loss of pancreatic beta cell function characterizing other forms of diabetes. ${ }^{10}$

In a recent US review of electronic hospital data, 11 of $32(34 \%)$ of admissions were not validated by endocrinologist review of biochemical and other data as DKA despite DKA being coded as the diagnosis. ${ }^{6}$ This is similar to the incorrect coding rates of $39.0 \%$ for initial episodes and $41.7 \%$ for recurrences in the present study, although we also had seven participants $(17.1 \%)$ with incomplete data for the initial episode. In most of these latter cases there was no documentation of plasma or urinary ketone concentrations, but other details required for diagnostic confirmation such as the plasma lactate (especially in metformin-treated participants with renal impairment ${ }^{31}$ ) may not have been available. These considerations highlight the necessity of medical record validation to correctly ascertain cases of DKA for healthcare quality assessment and planning purposes. In a similar vein, coding of type of diabetes in cases of DKA in the US study was incorrect in one in six cases. ${ }^{6}$ There are data suggesting that individuals with type 2 diabetes and DKA require higher daily total insulin doses, larger replacement fluid volumes and greater potassium supplementation to resolve their DKA than are used in type 1 diabetes. ${ }^{5}$ Accurate ascertainment of the type of diabetes could, therefore, influence the nature of initial management, although the effect of more intensive fluid resuscitation and insulin administration on prognosis in DKA complicating type 2 diabetes remains unknown.

The present study had limitations. The number of DKA episodes was restricted by the FDS2 cohort size and follow-up period but it was similar to those in comparable published studies with accurate ascertainment of $\operatorname{cases}^{6}$ and we employed more stringent $p$ values than usual to allow for the low numbers of DKA hospitalizations. Apart from mortality and changes in diabetes management, we did not record details of clinical outcomes including after discharge. As acknowledged, the time period of the study meant that DKA associated with SGLT2Is was not captured, with our definition of DKA excluding euglycemic cases. Lower serum glucose thresholds for DKA diagnosis have been recommended, such as $>11 \mathrm{mmol} / \mathrm{L}$ in the case of International Society for Paediatric and Adolescent Diabetes guidelines, ${ }^{32}$ but their use would not have changed case ascertainment in the present series. The strengths of the study are the community-based nature of the FDS2 cohort, and the use of detailed FDS2 and WADLS data, together with individual hospital case records, to determine diabetes type and ascertain DKA events.

The present study provides relatively robust estimates of the incidence of validated first DKA events in a representative cohort of Australians with well-characterized diabetes and its sequelae. The incidence rates of DKA complicating type 1 and 2 diabetes (which represented around three-quarters of all confirmed/probable cases) were generally within the ranges reported by other authors, while that for LADA was between those for type 1 and 2. These data are consistent with the strong independent inverse association between serum C-peptide (which can be measured as a marker of pancreatic beta cell function) and first DKA attendance. Participants with secondary diabetes, who were few in number but contributed disproportionately to the overall incidence, may be a group in which the risk of DKA could be underestimated.

Acknowledgements The authors would like to thank the patients and FDS staff for their involvement in the study, PathWest Laboratory Medicine at Fremantle Hospital for laboratory tests, and the staff at the Western Australian Data Linkage Branch, the Hospital Morbidity Data Collection, Emergency Department Data Collection and Death Registrations for data linkage. The authors would also like to thank the St John Ambulance Database for additional data linkage.

Funding The FDS2 was funded by National Health and Medical Research Council project grants 513781 and 1042231 . TMED is supported by a Medical Research Future Fund Practitioner Fellowship.

Competing interests None declared.

\section{Patient consent for publication Not required.}

Ethics approval The Fremantle Diabetes Study Phase II was approved by the Human Research Ethics Committee of the Southern Metropolitan Area Health Service.

Provenance and peer review Not commissioned; externally peer reviewed.

Data availability statement Data are available on reasonable request. The datasets generated during and/or analyzed during the current study are available from the corresponding author on request.

Open access This is an open access article distributed in accordance with the Creative Commons Attribution Non Commercial (CC BY-NC 4.0) license, which permits others to distribute, remix, adapt, build upon this work non-commercially, and license their derivative works on different terms, provided the original work is properly cited, appropriate credit is given, any changes made indicated, and the use is non-commercial. See: http://creativecommons.org/licenses/by-nc/4.0/.

ORCID iD

Timothy M E Davis http://orcid.org/0000-0003-0749-7411

\section{REFERENCES}

1 Fayfman M, Pasquel FJ, Umpierrez GE. Management of hyperglycemic crises: diabetic ketoacidosis and hyperglycemic hyperosmolar state. Med Clin North Am 2017;101:587-606.

2 Laffel L. Ketone bodies: a review of physiology, pathophysiology and application of monitoring to diabetes. Diabetes Metab Res Rev 1999;15:412-26.

3 Barski L, Nevzorov R, Jotkowitz A, et al. Comparison of diabetic ketoacidosis in patients with type-1 and type-2 diabetes mellitus. Am J Med Sci 2013;345:326-30.

$4 \mathrm{Xu} \mathrm{Y,} \mathrm{Bai} \mathrm{J,} \mathrm{Wang} \mathrm{G,} \mathrm{et} \mathrm{al.} \mathrm{Clinical} \mathrm{profile} \mathrm{of} \mathrm{diabetic} \mathrm{ketoacidosis} \mathrm{in}$ tertiary hospitals in China: a multicentre, clinic-based study. Diabet Med 2016;33:261-8.

5 Kamata Y, Takano K, Kishihara E, et al. Distinct clinical characteristics and therapeutic modalities for diabetic ketoacidosis in type 1 and type 2 diabetes mellitus. J Diabetes Complications 2017;31:468-72. 
6 VanderWeele J, Pollack T, Oakes DJ, et al. Validation of data from electronic data Warehouse in diabetic ketoacidosis: caution is needed. J Diabetes Complications 2018;32:650-4.

7 Fourlanos S, Dotta F, Greenbaum CJ, et al. Latent autoimmune diabetes in adults (LADA) should be less latent. Diabetologia 2005;48:2206-12.

8 Nabhan F, Emanuele MA, Emanuele N. Latent autoimmune diabetes of adulthood. unique features that distinguish it from types 1 and 2 . Postgrad Med 2005;117:7-12.

9 Djekic K, Mouzeyan A, Ipp E. Latent autoimmune diabetes of adults is phenotypically similar to type 1 diabetes in a minority population. J Clin Endocrinol Metab 2012;97:E409-13.

10 Chambers C, Fouts A, Dong F, et al. Characteristics of maturity onset diabetes of the young in a large diabetes center. Pediatr Diabetes 2016;17:360-7.

11 Larsen S. Diabetes mellitus secondary to chronic pancreatitis. Dan Med Bull 1993;40:153-62.

12 Maahs DM, Hermann JM, Holman N, et al. Rates of diabetic ketoacidosis: international comparison with 49,859 pediatric patients with type 1 diabetes from England, Wales, the U.S., Austria, and Germany. Diabetes Care 2015;38:1876-82.

13 Seidu S, Davies MJ, Mostafa S, et al. Prevalence and characteristics in coding, classification and diagnosis of diabetes in primary care. Postgrad Med J 2014;90:13-17.

14 Davis TME, Bruce DG, Davis WA. Cohort profile: the Fremantle diabetes study. Int J Epidemiol 2013;42:412-21.

15 Levey AS, Stevens LA, Schmid CH, et al. A new equation to estimate glomerular filtration rate. Ann Intern Med 2009;150:604-12

16 Feldman EL, Stevens MJ, Thomas PK, et al. A practical two-step quantitative clinical and electrophysiological assessment for the diagnosis and staging of diabetic neuropathy. Diabetes Care 1994;17:1281-9.

17 Davis WA, Peters KE, Makepeace A, et al. Prevalence of diabetes in Australia: insights from the Fremantle diabetes study phase II. Intern Med J 2018;48:803-9.

18 Holman C D'Arcy J, Bass AJ, Rosman DL, et al. A decade of data linkage in Western Australia: strategic design, applications and benefits of the WA data linkage system. Aust Health Rev 2008;32:766-77.

19 Fazeli Farsani S, Brodovicz K, Soleymanlou N, et al. Incidence and prevalence of diabetic ketoacidosis (DKA) among adults with type 1 diabetes mellitus (T1D): a systematic literature review. BMJ Open 2017;7:e016587.
20 Erondu N, Desai M, Ways K, et al. Diabetic ketoacidosis and related events in the canagliflozin type 2 diabetes clinical program. Diabetes Care 2015;38:1680-6.

21 Scheen AJ. An update on the safety of SGLT2 inhibitors. Expert Opin Drug Saf 2019;18:295-311.

22 Hamblin PS, Wong R, Ekinci El, et al. Sglt2 inhibitors increase the risk of diabetic ketoacidosis developing in the community and during hospital admission. J Clin Endocrinol Metab 2019;104:3077-87.

23 Jensen ML, Persson F, Andersen GS, et al. Incidence of ketoacidosis in the Danish type 2 diabetes population before and after introduction of sodium-glucose cotransporter 2 Inhibitors-A nationwide, retrospective cohort study, 1995-2014. Diabetes Care 2017;40:e57-8.

24 Kim Y-G, Jeon JY, Han SJ, et al. Sodium-Glucose co-transporter-2 inhibitors and the risk of ketoacidosis in patients with type 2 diabetes mellitus: a nationwide population-based cohort study. Diabetes Obes Metab 2018;20:1852-8.

25 Australian Diabetes Society. Severe euglycaemic ketoacidosis with SGLT2 inhibitor use in the perioperative period, 2018. Available: https://diabetessociety.com.au/documents/2018_ALERT-ADS SGLT2i_PerioperativeKetoacidosis_v3_final2018_02_14.pdf [Accessed June 2019].

26 Davis TME, Brown SGA, Jacobs IG, et al. Determinants of severe hypoglycemia complicating type 2 diabetes: the Fremantle diabetes study. J Clin Endocrinol Metab 2010;95:2240-7.

27 Henderson JN, Allen KV, Deary IJ, et al. Hypoglycaemia in insulintreated type 2 diabetes: frequency, symptoms and impaired awareness. Diabet Med 2003;20:1016-21.

28 Hernandez M, Mollo A, Marsal JR, et al. Insulin secretion in patients with latent autoimmune diabetes (LADA): half way between type 1 and type 2 diabetes: action LADA 9. BMC Endocr Disord 2015;15:1.

29 Wibell L, Nyström L, Ostman J, et al. Increased mortality in diabetes during the first 10 years of the disease. A population-based study (diss) in Swedish adults 15-34 years old at diagnosis. J Intern Med 2001;249:263-70.

30 Guillausseau PJ, Massin P, Dubois-LaForgue D, et al. Maternally inherited diabetes and deafness: a multicenter study. Ann Intern Med 2001;134:721-8.

31 Lazarus B, Wu A, Shin J-I, et al. Association of metformin use with risk of lactic acidosis across the range of kidney function: a community-based cohort study. JAMA Intern Med 2018;178:903-10.

32 Wolfsdorf Jl, Glaser N, Agus M, et al. ISPAD clinical practice consensus guidelines 2018: diabetic ketoacidosis and the hyperglycemic hyperosmolar state. Pediatr Diabetes 2018;19 Suppl 27:155-77. 